\title{
Flood Early Warning System Design Using Wireless Sensor Network
}

\author{
E Madona $^{1}$, Y Yulastri², M Irmansyah ${ }^{3}$, A Nasution $^{4}$ \\ Politeknik Negeri Padang, Kampus Politeknik Negeri Padang-Limau manis, \\ Padang,Indonesia ${ }^{1,2,3,4}$ \\ \{emadona38@gmail.com $\left.{ }^{1}\right\}$
}

\begin{abstract}
This research designs and implements wireless sensor networks as a tool for delivery, routers and data recipients at flood early warning in batu busuk river, Padang - West Sumatera. The multihop wireless communication system using XBee Pro S2 with one of two routers acts as a backup communication path. The system will consist of end devices include humidity temperature sensor, rainfall, wind direction, and wind speed, Arduino Uno, and XBee S2, 2 XBee S2 router, and a coordinator covering XBee S2 and raspberry Pi as database server. The test results show the minimum network recovery time required by the device to build a new communication network after the network was disconnected due to router off is 7,5 seconds and maximum time is 84 seconds. Data packet loss got $0 \%$ at $100 \mathrm{~m}$ to $150 \mathrm{~m}$ and $1 \%$ with $250 \mathrm{~m}$. The process of self-healing networks can work well and the length of time required by the end device to perform the manufacture of communication networks is 10.5 seconds. Testing the delay of sending data from end device to database server is $63,6 \mathrm{~s}$.
\end{abstract}

Keywords: Arduino Uno, Database server, Rainfall Sensor, Wireless Sensor Network, XBee2.

\section{Introduction}

Indonesia has seasons with an extraordinary shift. Usually dry periods of April-September and the rainy season in October-March with the transition seasons in March / April and September / October. But today the season changes are uncertain. The changing weather conditions can cause disasters such as floods [1], [2], and also a flood in Padang West Sumatra. According to BNPB data 2016, the area of Extreme Weather Catastrophe (Landslides and Flood) in West Sumatra Province, Padang city in the Middle - High category. This category has medium to high potential for land movement, floods, and landslides. These conditions can occur if the rainfall above normal, especially in areas adjacent to river valleys, escarpments, and road cliffs. Flood monitoring that has been done mostly depends on the infrastructure around the disaster site, such as the availability of electricity and communication networks, so the construction of disaster monitoring sites is limited by the availability of these facilities. Monitoring of flood is usually done in the river upstream which is not only far from the settlement but also from the facilities. Therefore, the use of wireless sensor networks is good solution. Wireless Sensor Network Technology (WSNs) has developed quite rapidly[3]. WSNs 
applications are now widely applied to forest fires[4], water quality[5], landslides [6] and floods[7]. Sunkpho's paper using start flow commercial tools to measure the water level, sensor readout data is saved to database server and displayed via web and sms[8]. Data delivery of each node in this study using GPRS module and there are 15 tools installed. This configuration course requires a high cost. In addition, the unstable GPRS connection makes sending data difficult. The use of WSNs for flood warning systems is also explained by[9]. In the research they use water level sensors and temperature, the sensing results are then transmitted using data logger and GPRS to be stored on the server, FTP receives data and stores in the folder specific from each station code to then display via website. Use of GPRS and FTP in this study has weaknesses. FTP design is still weak so it does not inform the system administrators about the risks they face, other than that FTP is not safe in transferring a file because the file sending without being encrypted first. In this research we developed a Wireless Sensor Network system design to monitoring the flood early warning system using XbeeS2 for sending and receiving sensor data readings [10], utilizing AODV routing protocol and network capability self healing on xbee [11] to maintain continuity of information to the database server with the tcp client communication protocol. The design of WSN system in this research is implemented for monitoring of flood early warning system in area Batubusuk, Padang - West Sumatera.

\section{System Design And Implementation}

\section{I System Architecture}

Generally speaking, the procedure of this sensor network is collecting data readings from the sensor on each node then communicated ad-hoc with other nodes until the data gets the data manager. Figure 1 illustrated the data sensor output transmission starts from the right side to the left side up to the yellow point then sent to the database server. Data from the right node is expected to communicate ad-hoc through the nodes next to the node coordinator via the router node.

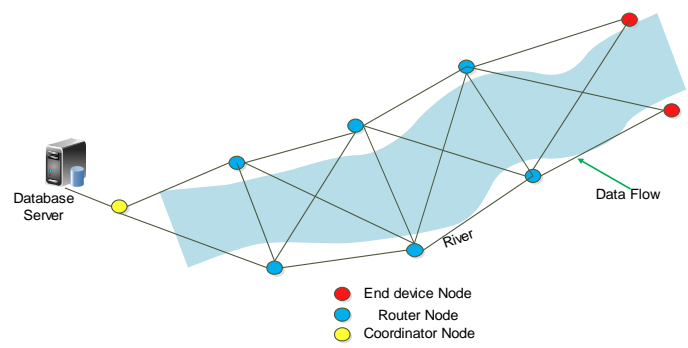

Figure 1. Wireless Sensor data acquisition architecture Network for monitoring flood early warning

\subsection{Design and Implementation of Node Sensor Devices}

In this paper, the sensor nodes are classified into three types of sensor nodes namely end devices, routers, and coordinators. Each node is equipped with a microcontroller, sensor, and $\mathrm{RF}$ module, except at the node coordinator. The readings of data sensor will be sent in the form of data packets. Packages sent from sensor nodes with the format as in Figure 2.

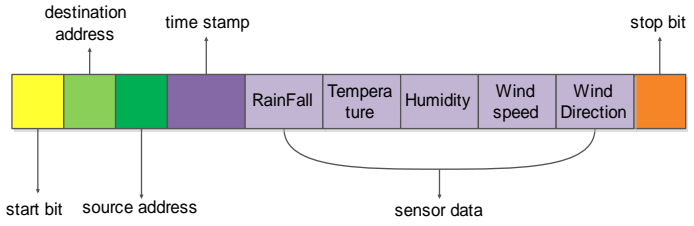


Figure 2. The packet format that exits the node

\subsubsection{End Device}

End device is the node at the end of the system. The node has only one task, which is forwarding the data packets from the sensor readout to router. The end device itself can choose the router to forward its data packet to the coordinator. The end device sends its data packet to the destination address is the node address of the router. If received by the router according to the destination address of the packet, the router sends the ACK to the end device as confirmation of the data has been received, and the end device will send through the router for the next data packet. In the End device uses 5 (five) sensors. The sensors will be processed by using a microcontroller that will be displayed in serial form, where the serial data will be sent using a wireless sensor network to the receiver.

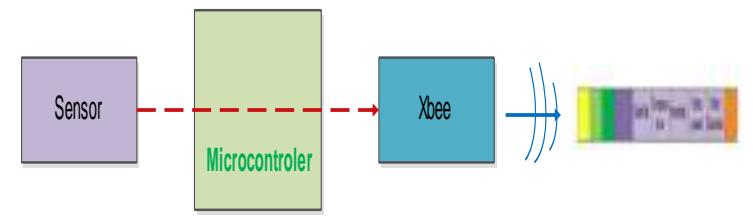

Figure 3. The flow of data from the sensor to be transmitted

\subsubsection{Router}

Once data is sent from the device it will be forwarded to the coordinator through the router because the distance between the end device and the coordinator is very far away. In the data routing process, the router is continuing to transfer the data packets to the destination address in the network. This function makes the router must always be active and require more power in operation. In this section, there are 2 routers, which have task to create a backup path if one of the routers is off or not working so the data transmission is not interrupted. Routers can also specify hops to be selected next. With the same steps as end device does, the router also waits for the ACK signal from the intended router to detect its routing. If it does not receive an ACK, the router also sends another packet to router until another router gives ACK signal. If the router can directly communicate with the coordinator, the router directly forwards the package coordinator without waiting for ACK signal to send the next packets because it considered a router can reach the coordinator. The data packet is definitely received by the coordinator even without ACK signal from the coordinator.

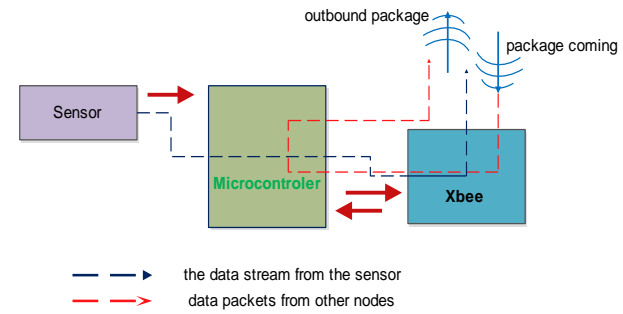

Figure 4. The work process of the device on the node 


\subsubsection{Coordinator}

The actual coordinator is the receiving node. So, the coordinator only receives data from the router that communicates and then passes the data to the computer. The coordinator is like a bridge that connects data from the router to the database server. In this section, data sent by end device through the router will be received by the coordinator via wireless in serial data. The coordinator itself consists of microcontroller, Ethernet shield, Xbee, RTC, and modem router. Data that has been received via XBee, will be sent back to raspberry pi which serves as server, sending itself using Ethernet shield using router internet modem. Figure 5 shows the data flow at the coordinator node

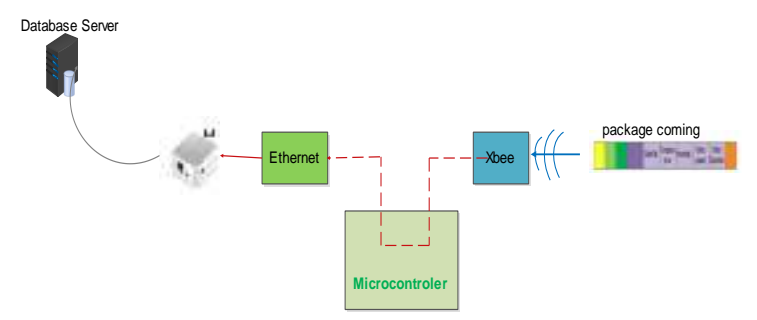

Figure 5. Data flow at node coordinator

\subsection{System Performance Measurement Scenario}

To analyze the performance of the previous system, measurements are carried out on the conditions that represent the implementation of the system created. Wireless sensor network built in this research is applied to monitoring early flood warning. Monitoring conducted on the river area Batubusuk Padang - West Sumatra. This measurement will be done using XBee Pro S2 modules, one module as the end device node, two modules on node routers, and the last module coordinator node. Ethernet shield is used for sending data to database server with SD card for data storage. The sending data will be added date and time of delivery information by the RTC circuit. So, it can inform how long data delay reaches the server and compare the time delay data transmission to server. Before doing this measurement it needs to measure the coverage area of Xbee Pro Series 2 module. The coverage area of RF module measurement is the area around the river Batubusuk. This measurement is expected the node coordinator can receive from three other nodes (one end device node, and two router nodes). The data received by the coordinator node can be used as a reference for the delay-end-to-end determination and packet loss of the system.

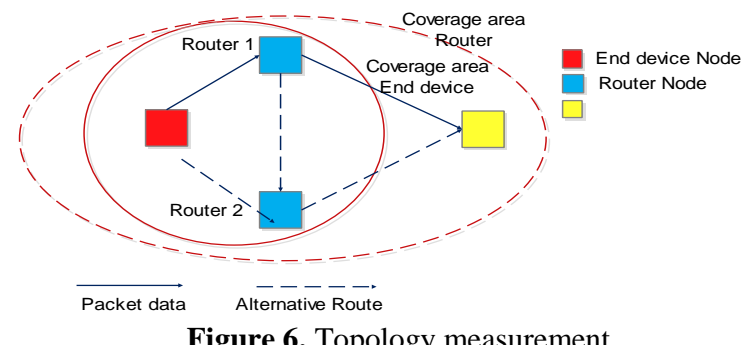

Figure 6. Topology measurement

In this paper, there are three tests to measure the time of data transmission: 
Testing 1 - Testing Delay Network Recovery with Distance Variation and packet loss, by changing the distance between devices to determine the effect of the distance to the time required for the process of making new communication networks. Observations started from $100 \mathrm{~m}$ to $400 \mathrm{~m}$ and sending 80 data packets with the condition of an outdoor observation location. The experiment begins by positioning between the coordinator and the end device cannot communicate directly, so router assistance is needed. The distance between the router and the coordinator is equal to the distance of the router with the end device. This experiment is done by turning router 1 and router 2 interchangeably. After the router is turned on alternately, the distance between devices plus 50 meters to see the effect of adding distance to network recovery time.

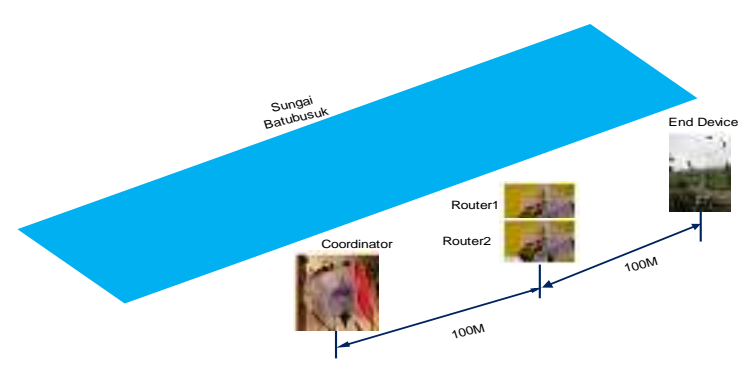

Figure 7. The Distance of Each Xbee Device

Testing 2- Routing Recovery Time Testing, this test to determine the length of time that the end device needs to establish a new communication path when the existing communication path is disconnected or can no longer be used. Testing is done by switching the router alternately at certain intervals with the distance from the end device to the router as far as $200 \mathrm{~m}$, so obtained the time interval needed for the process of switching from a broken communication network to a new communication path.

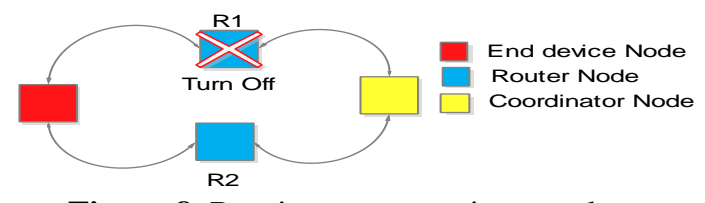

Figure 8. Routing recovery time test layout

Testing 3- Measurement of Delay into Server, this test is done to know the delay from coordinator to database server. For that required circuit RTC (Real-time Clock) in order to compare the hours of data coming in the database server

\section{RESULT AND DISCUSSION}

\subsection{Test Delay Network Recovery with Distance Variation and Packet Loss}

Table 1 shows the length of time required for the process of establishing a new communication network when the router is turned off. Column Time 1 represents the delay network recovery experienced by the network bypassing router 1 , and Time 2 represents the delay network recovery experienced by the network bypassing router 2 . From table 1 it can be 
seen that the network recovery time gained increased following the increase of the distance between devices.

Table 1: DNR and Packet loss testing with Distance Variation

\begin{tabular}{cllllll}
\hline No & $\begin{array}{l}\text { End device- } \\
\text { Router 1- } \\
\text { Coordinator } \\
(\mathrm{m})\end{array}$ & Time 1 & $\begin{array}{c}\% \\
\text { Packet } \\
\text { loss }\end{array}$ & $\begin{array}{l}\text { End device- } \\
\text { Router2- } \\
\text { Coordinator } \\
(\mathrm{m})\end{array}$ & $\begin{array}{c}\text { Time } \\
2\end{array}$ & $\begin{array}{c}\% \\
\text { Packet } \\
\text { loss }\end{array}$ \\
\hline 1 & $100-100$ & 7.5 & 0 & $100-100$ & 9 & 0 \\
\hline 2 & $150-150$ & 10 & 0 & $150-150$ & 13 & 0 \\
\hline 3 & $200-200$ & 12 & 1 & $200-200$ & 15 & 1 \\
\hline 4 & $250-250$ & 15 & 1 & $250-250$ & 17.5 & 2 \\
\hline 5 & $300-300$ & 27.5 & 5 & $300-300$ & 31 & 5 \\
\hline 6 & $350-350$ & 75 & 8 & $350-350$ & 84 & 10 \\
\hline 7 & $400-400$ & $\begin{array}{l}\text { No } \\
\text { Signal }\end{array}$ & $\begin{array}{l}\text { No } \\
\text { signal }\end{array}$ & $400-400$ & $\begin{array}{l}\text { No } \\
\text { signal }\end{array}$ & $\begin{array}{l}\text { No } \\
\text { signal }\end{array}$ \\
\hline
\end{tabular}

Based on the measurement result, the distance of 100 meters up to 350 meters, the time required for the recovery process has a minimum value of 7.5 seconds and a maximum delay of 84 seconds. However, for a distance of $400 \mathrm{~m}$ from End device to the Router and $400 \mathrm{~m}$ from the router to the communication coordinator disconnected because exceeding maximum module distance capability. For packet loss, at a distance of 100 to $150 \mathrm{~m}$ at $0 \%$ and at a distance of 250 $\mathrm{m}$ by $1 \%$, this is in accordance with research [10]. The above experiment can be attributed to the data transmission delay, ie the time it takes data to arrive from the transmitter to the receiver. In the transmission of communication in a medium, when the wave of information propagates it will take time, this propagation time that makes the sending time is not equal to the time received. It can be concluded that the further distance between the communications devices used, the longer the information it takes to get to the receiver.

\subsection{Routing Recovery Time Testing}

Table 2 shows the length of time to create new communication lines (delay network recovery). From table 2 can be concluded that the process of self-healing network can work well and the length of time required by end device to make the communication network from 6 experiments, with an average of 10.5 seconds. Tabel 2: Routing recovery time testing

Tabel 2: Routing recovery time testing

\begin{tabular}{|c|c|c|c|}
\hline \multirow{2}{*}{ No } & \multicolumn{2}{|c|}{ Router } & \multirow{2}{*}{ Delay(s) } \\
\hline & $\mathrm{R} 1$ & $\mathrm{R} 2$ & \\
\hline 1 & Off & On & 14 \\
\hline 2 & On & Off & 10 \\
\hline 3 & Off & On & 10 \\
\hline 4 & On & Off & 9 \\
\hline 5 & Off & On & 9 \\
\hline 6 & On & Off & 11 \\
\hline \multicolumn{3}{|c|}{ Average } & 10,5 \\
\hline
\end{tabular}

This result is much different from the research [11], this is due to the difference in distance between the end device to router 1 and router 2 . 


\subsection{Testing Delay Into the Server Database}

Table 3: Testing the sender time to the server

\begin{tabular}{|c|c|c|c|}
\hline No & $\begin{array}{l}\text { RTC } \\
\text { (hh:mm:ss) }\end{array}$ & $\begin{array}{l}\text { Server } \\
\text { (hh:mm:ss) }\end{array}$ & $\begin{array}{l}\text { Delay } \\
\text { (ss) }\end{array}$ \\
\hline 1 & $17: 24: 35$ & $17: 25: 41$ & 66 \\
\hline 2 & $17: 24: 42$ & $17: 25: 46$ & 64 \\
\hline 3 & $17: 24: 45$ & $17: 25: 49$ & 64 \\
\hline 4 & $17: 24: 44$ & $17: 25: 47$ & 63 \\
\hline 5 & $17: 24: 47$ & $17: 25: 50$ & 63 \\
\hline 6 & $17: 24: 49$ & $17: 25: 52$ & 63 \\
\hline 7 & $17: 24: 51$ & $17: 25: 54$ & 63 \\
\hline \multirow[t]{2}{*}{8} & $17: 24: 53$ & $17: 25: 56$ & 63 \\
\hline & & & 63,6 \\
\hline
\end{tabular}

From 8 test results 8 data from time from Coordinator to server average 63,6 second or 1 minute 3 second. It can be concluded that the data displayed updates and realtime due to the time difference is relatively small.

\section{Conclusions}

The minimum network recovery time required by the device to build a new communication network after the network is disconnected caused router off is 7,5 seconds and maximum 84 seconds with distance $100 \mathrm{~m}$ to $350 \mathrm{~m}$ and packet loss of $1 \%$ to $250 \mathrm{~m}$ distance. The effect of distance to data reception time is directly proportional. The farther the distance between the end device, the router and the coordinator, the longer the data reception time. Routing recovery time result is 10,5 second and testing delay of data delivery from end device to raspberry pi server is $63,6 \mathrm{~s}$.

\section{References}

[1] R. T. Wetherald, "Simulation of hydrologic changes associated with global warming," J. Geophys. Res., vol. 107, no. D19, p. 4379, 2002.

[2] M. K. Lindell and C. S. Prater, "Assessing community impacts of natural disasters," Nat. hazards Rev., vol. 4, no. 4, pp. 176-185, 2003.

[3] V. J. Hodge, S. O'Keefe, M. Weeks, and A. Moulds, "Wireless sensor networks for condition monitoring in the railway industry: A survey," IEEE Trans. Intell. Transp. Syst., vol. 16, no. 3, pp. 1088-1106, 2014.

[4] G. Wang, J. Zhang, W. Li, D. Cui, and Y. Jing, "A forest fire monitoring system based on GPRS and ZigBee wireless sensor network," in 2010 5th IEEE Conference on Industrial Electronics and Applications, 2010, pp. 1859-1862.

[5] Z. Rasin and M. R. Abdullah, "Water quality monitoring system using zigbee based wireless sensor network," Int. J. Eng. Technol., vol. 9, no. 10, pp. 24-28, 2009.

[6] A. Rosi et al., "Landslide monitoring with sensor networks: experiences and lessons learnt from a real-world deployment," Int. J. Sens. Networks, vol. 10, no. 3, pp. 111$122,2011$.

[7] M. A. Islam, T. Islam, M. A. Syrus, and N. Ahmed, "Implementation of flash flood monitoring system based on wireless sensor network in Bangladesh," in 2014 
International Conference on Informatics, Electronics \& Vision (ICIEV), 2014, pp. 1-6.

[8] J. Sunkpho and C. Ootamakorn, "Real-time flood monitoring and warning system.," Songklanakarin J. Sci. Technol., vol. 33, no. 2, 2011.

[9] S. Keoduangsine and R. Goodwin, "A GPRS-based data collection and transmission for flood warning system: The case of the Lower Mekong River Basin," Int. J. Innov. Manag. Technol., vol. 3, no. 3, p. 217, 2012.

[10] A. Cama-Pinto, G. Pineres-Espitia, J. Caicedo-Ortiz, E. Ramírez-Cerpa, L. BetancurAgudelo, and F. Gómez-Mula, "Received strength signal intensity performance analysis in wireless sensor network using Arduino platform and XBee wireless modules," Int. J. Distrib. Sens. Networks, vol. 13, no. 7, p. $1550147717722691,2017$.

[11] R. Piyare and S. Lee, "Performance analysis of XBee ZB module based wireless sensor networks,” Int. J. Sci. Eng. Res., vol. 4, no. 4, pp. 1615-1621, 2013. 